\title{
An Existence Result for a Class of Extended Inclusion Problems with Applications to Equilibrium Problems
}

\author{
Y. P. Fang and N. J. Huang
}

\begin{abstract}
Let $X$ be a real reflexive Banach space, $K \subset X$ a nonempty, closed and convex set, and $F: K \times K \rightarrow 2^{X}$ (the family of all the subsets of $X$ ) be a multi-valued mapping. In this paper, we consider the following extended inclusion problem: find $x^{*} \in K$ such that $K \subset F\left(x^{*}, x^{*}\right)$. Under suitable conditions, we establish an existence result for the extended inclusion problem. As applications, we give some existence theorems for equilibrium problems.
\end{abstract}

Keywords. Extended inclusion problem, equilibrium problem, variational inequality, existence

Mathematics Subject Classification (2000). Primary 49J40, secondary 47H04

\section{Introduction and Preliminaries}

Given a set $K$ and a bifunction $f: K \times K \rightarrow R$, the equilibrium problem is to find $x \in K$ such that

$$
f(x, y) \geq 0 \quad \forall y \in K
$$

which was introduced by Blum and Oettli [5]. The equilibrium problem has been studied extensively because it provides a unifying framework for many important problems, such as optimization, variational and variational-like inequalities, minimax inequality problems. They are widely applied to study the problems arising in economics, mechanics, and engineering science. In recent years, many existence results concerning equilibrium problems and variational inequalities problems have been established by different methods (see, for example, [1, 2], [4]-[8], [10]-[12], [14]-[16], [19] and the references therein). Recently, Di Bella [9] introduced and studied a class of inclusion problems, which is formulated to find $x^{*} \in K$ such that $K \subset T\left(x^{*}\right)$, where $K$ is a nonempty,

Ya-ping Fang: Department of Mathematics, Sichuan University, Chengdu, Sichuan 610064, P. R. China, Corresponding author; fabhcn@yahoo.com.cn

Nan-jing Huang: Department of Mathematics, Sichuan University, Chengdu, Sichuan 610064, P. R. China 
closed and convex subset of a real Banach space $X$, and $T: K \rightarrow 2^{X}$ is a multi-valued mapping. He obtained a general existence result for the inclusion problem, which extends the corresponding result by Ricceri [19]. Di Bella [9] also pointed out that the inclusion problem includes the variational inequality problem as a special case. In fact, the equilibrium problem also can be regarded as a special case of the above inclusion problem by defining $T: K \rightarrow 2^{X}$ by

$$
T(x)=\{y \in X: f(x, y) \geq 0\} \quad \forall x \in K .
$$

In this paper, we further introduce and study the extended inclusion problem, which is to find $x^{*} \in K$ such that $K \subset F\left(x^{*}, x^{*}\right)$, where $F: K \times K \rightarrow 2^{X}$ is a multi-valued mapping. The extended inclusion problem provides a unifying framework for the extended variational inequality problem introduced by Chen [8], some variational-like problems studied in $[1,7]$, the extended equilibrium problem in [10] and the inclusion problem introduced by Di Bella [9]. Under suitable conditions, we prove the solvability of the extended inclusion problem. As applications, we give some existence results for equilibrium problems and variational inequality problems.

Given two real Banach spaces $X$ and $Y$, and a multi-valued mapping $T$ : $X \rightarrow 2^{Y} . T$ is said to be lower semi-continuous if, for each open set $\Omega \subset Y$, the set $T^{-}(\Omega)=\{x \in X: T(x) \cap \Omega \neq \emptyset\}$ is open in $X$. $T$ is said to be upper semi-continuous if, for each closed set $A \subset Y$, the set $T^{-}(A)$ is closed in $X$. T is said to have a closed graph if the set $\operatorname{Graph}(T)=\{(x, y) \in X \times Y: y \in T(x)\}$ is closed in $X \times Y$. When $\mathcal{R}(T)=\{y \in Y: y \in T(x)$ for some $x \in X\}$ is contained in a compact subset of $Y$, it is known that $T$ is upper semi-continuous if and only if $T$ has a closed graph (see [3]).

The paper is organized as follows: In Section 2, we give our main result by establishing an existence result for the extended inclusion problem. As applications of our result, we give some existence results for equilibrium problems.

\section{Existence results}

For our main result, we first present an existence result for the inclusion problem.

Lemma 2.1. Let $K$ be a nonempty, compact and convex subset of a finite dimensional Banach space $X$ and $T: K \rightarrow 2^{X}$ be a multi-valued mapping satisfying the following conditions:

(i) $x \in T(x)$ for all $x \in K$;

(ii) the multi-valued mapping $T^{c}: K \rightarrow 2^{X}$ defined by $T^{c}(x)=X \backslash T(x)$ is lower semi-continuous with convex values.

Then there exists $x^{*} \in K$ such that $K \subset T\left(x^{*}\right)$. 
Proof. Define $T_{K}^{c}: K \rightarrow 2^{K}$ by $T_{K}^{c}(x)=K \cap T^{c}(x)=K \backslash T(x)$. We claim that there exists $x^{*} \in K$ such that $T_{K}^{c}\left(x^{*}\right)=\emptyset$. Assume by absurd that $T_{K}^{c}(x) \neq \emptyset$ for all $x \in K$. Condition (ii) implies that $T_{K}^{c}$ is lower semi-continuous with nonempty and convex values. By the Michael selection theorem [13], there exists a continuous single-valued mapping $g: K \rightarrow K$ with $g(x) \in T_{K}^{c}(x)$ for all $x \in K$. By the Brouwer fixed point theorem, there exists $u \in K$ such that $u=g(u) \in K \backslash T(u)$, which contradicts condition (i). Hence there exists $x^{*} \in K$ such that $T_{K}^{c}\left(x^{*}\right)=\emptyset$. So follows the conclusion.

Remark 2.2. Some existence results for the inclusion problem were proved in Theorems 3.1 and 3.2 of Di Bella in [9]. But our assumption is different and the proof is more simple.

Now we present our main result as follow:

Theorem 2.3. Let $K$ be a nonempty, bounded, closed and convex subset of a real reflexive Banach space $X$ and $F: K \times K \rightarrow 2^{X}$ be a multi-valued mapping. Assume that the following conditions hold:

(i) $x \in F(y, x)$ for all $x, y \in K$;

(ii) for each $z \in K$ and each finite dimensional subspace $D$ of $X$ with $K_{D}=$ $K \cap K \neq \emptyset$, the multi-valued mapping $F^{c}(z, \cdot): K_{D} \rightarrow 2^{X}$ is lower semicontinuous with convex values;

(iii) for each $z \in K$ and each finite dimensional subspace $D$ of $X$ with $K_{D} \neq \emptyset$, the set $\left\{x \in K_{D}: K_{D} \subset F(z, x)\right\}$ is convex and closed;

(iv) if $\left(x_{\alpha}, z_{\alpha}\right) \in K \times K,\left(x_{\alpha}, z_{\alpha}\right)$ converges to $(x, z) \in K \times K$ weakly, and $K \subset F\left(z_{\alpha}, x_{\alpha}\right)$ for all $\alpha$, then $K \subset F(z, x)$.

Then there exists $x^{*} \in K$ such that $K \subset F\left(x^{*}, x^{*}\right)$.

Proof. Define $\mathcal{D}$ by

$$
\mathcal{D}=\left\{D: D \text { is a finite dimensional subspace of } X \text { with } K_{D} \neq \emptyset\right\} .
$$

For any given $z \in K$ and $D \in \mathcal{D}$, consider the following auxiliary problem:

$(\mathrm{AP})_{D}^{z}: \quad$ Find $u \in K_{D}$ such that $K_{D} \subset F(z, u)$.

Conditions (i) and (ii) imply that for each $z \in K, F(z, \cdot)$ satisfies all the conditions of Lemma 2.1. By Lemma 2.1, problem $(A P)_{D}^{z}$ is solvable for all $z \in K$ and $D \in \mathcal{D}$. Define a multi-valued $T: K_{D} \rightarrow 2^{K_{D}}$ as follows:

$$
T(z)=\left\{u \in K_{D}: u \text { solves problem }(\mathrm{AP})_{D}^{z}\right\} \quad \forall z \in K_{D} .
$$

By the arguments above and condition (iii), it is easy to verify that $T(z)$ is nonempty, closed, and convex for all $z \in K_{D}$. Now we show that $T$ is also upper semi-continuous. Let $\left(z_{\alpha}, u_{\alpha}\right) \in \operatorname{Graph}(T)$ and $\left(z_{\alpha}, u_{\alpha}\right)$ converge to $(z, u)$ 
with respect to the norm topology of $X \times X$. It follows from condition (iv) that $(z, u) \in \operatorname{Graph}(T)$. Thus $T$ has a closed graph and so is upper semi-continuous. By the known Kakutani-Fan-Glicksberg fixed point theorem (see [13, p. 171]), there exists $u^{*} \in K_{D}$ such that $K_{D} \subset F\left(u^{*}, u^{*}\right)$. For any $D \in \mathcal{D}$, denote by $S_{D}$ the solution set of the following problem:

$$
\text { Find } u \in K \text { such that } K_{D} \subset F(u, u) \text {. }
$$

Obviously, $S_{D}$ is nonempty and bounded for all $D \in \mathcal{D}$. Denote by $\bar{S}_{D}^{w}$ the weak closure of $S_{D}$ in $K$. Clearly $\bar{S}_{D}^{w}$ is weakly compact. For any $D_{i} \in \mathcal{D}, i=1, \ldots, n$, let $L$ be the subspace spanned by $\bigcup_{i=1}^{n} D_{i}$. It is easy to see that $S_{L} \subset S_{D_{i}}$ since $D_{i} \subset L$ for each $i$. Thus $S_{L} \subset \bigcap_{i=1}^{n} S_{D_{i}}$. This implies that $\left\{\bar{S}_{D}^{w}: D \in \mathcal{D}\right\}$ has a finite intersection property. It follows from [17, Theorem 1.2.3] that

$$
\bigcap_{D \in \mathcal{D}} \bar{S}_{D}^{w} \neq \emptyset
$$

Let $x^{*} \in \bigcap_{D \in \mathcal{D}} \bar{S}_{D}^{w}$. We assert that $K \subset F\left(x^{*}, x^{*}\right)$. In fact, for any given $D \in \mathcal{D}$, there exists $\left\{x_{\alpha}\right\} \subset S_{D}$ such that $x_{\alpha}$ converges to $x^{*}$ weakly since $x^{*} \in \bar{S}_{D}^{w}$. It follows that for any given $D \in \mathcal{D}, K_{D} \subset F\left(x_{\alpha}, x_{\alpha}\right)$. Condition (4) implies that $K_{D} \subset F\left(x^{*}, x^{*}\right)$ for all $D \in \mathcal{D}$. Therefore, $K=\bigcup_{D \in \mathcal{D}} K_{D} \subset F\left(x^{*}, x^{*}\right)$. The proof is complete.

Remark 2.4. When $F(z, x) \equiv F(x)$, we can obtain a corresponding existence result for the inclusion problem.

\section{Applications}

In this section, we shall apply Theorem 2.3 to present the solvability of the extended equilibrium problem. In what follows, unless otherwise specified, we always let $X$ be a real reflexive Banach space, $K \subset X$ be a nonempty, bounded, closed, and convex set. For our further results, we recall some concepts and lemmas.

Definition 3.1. A bifunction $f: K \times K \rightarrow R$ is said to be pseudomonotone if, for any $x, y \in K, f(x, y) \geq 0$ implies $f(y, x) \leq 0$.

Definition 3.2. A bifunction $f: K \times K \rightarrow R$ is said to be hemicontinuous if, for any given $x, y \in K$, the mapping $t \mapsto f(x+t(y-x)), y)$ is continuous at $0^{+}$.

Definition 3.3. A function $g: K \rightarrow R$ is said to be completely continuous if $g$ is continuous with respect to the weak topology of $X$.

To obtain the existence of the extended equilibrium problem, we need the following lemmas which can be found in $[4,5]$. 
Lemma 3.4 (see $[4,5]$ ). Let $K$ be a nonempty convex set, $x_{0} \in K$ a given point, and $f: K \times K \rightarrow R$ be a hemicontinuous and pseudomonotone bifunction satisfying the following conditions:

(i) $f(x, x) \geq 0$ for all $x \in K$;

(ii) If $x, y, z \in K, f(x, y) \leq 0$, and $f(x, z)<0$, then $f(x, t y+(1-t) z)<0$ for all $0<t<1$.

Then the following are equivalent:

(I) $f\left(x_{0}, y\right) \geq 0$ for all $y \in K$;

(II) $f\left(y, x_{0}\right) \leq 0$ for all $y \in K$.

Lemma 3.5 (see $[4,5]$ ). Let $K$ be a nonempty, bounded, closed and convex subset of $X$ and $f: K \times K \rightarrow R$ be a hemicontinuous pseudomonotone bifunction satisfying the following conditions:

(i) $f(x, x) \geq 0$ for all $x \in K$;

(ii) if $x, y, z \in K, f(x, y) \leq 0$, and $f(x, z)<0$, then $f(x$, ty $+(1-t) z)<0$ for all $0<t<1$;

(iii) for any given $x \in K, f(x, \cdot)$ is convex;

(iv) for any given $x \in K, f(x, \cdot)$ is continuous.

Then there exists $x^{*} \in K$ such that $f\left(x^{*}, z\right) \geq 0$ for all $z \in K$. In addition, the solution set is bounded, closed and convex.

Now we apply Theorem 2.3 and the above lemmas to prove the solvability of a class of extended equilibrium problems.

Theorem 3.6. Let $K$ be a nonempty, bounded, closed, convex subset of a real reflexive Banach space $X$, and $\varphi: K \times K \times K \rightarrow R$ be a function satisfying the following conditions:

(1) for any given $u \in K, \varphi(u, \cdot, \cdot)$ is pseudomonotone;

(2) for any fixed $u, w \in K$ and fixed finite dimensional subspace $D \subset X$ with $K_{D} \neq \emptyset, \varphi(u, \cdot, v): K_{D} \rightarrow R$ is continuous;

(3) $\varphi(u, v, v) \geq 0$ for all $u, v \in K$;

(4) if $u, x, y, z \in K, \varphi(u, x, y) \leq 0$ and $\varphi(u, x, z)<0$, then $\varphi(u, x, t y+(1-t) z)<0$ for all $0<t<1$

(5) for any given $u, x \in K, \varphi(u, x, \cdot)$ is convex;

(6) for any given $v \in K, \varphi(\cdot, v, \cdot)$ is completely continuous.

Then there exists $x^{*} \in K$ such that $\varphi\left(x^{*}, x^{*}, y\right) \geq 0$ for all $y \in K$.

Proof. Define a multi-valued map $F: K \times K \rightarrow 2^{X}$ as follows:

$$
F(z, x)=\{y \in X: \varphi(z, x, y) \geq 0\} \quad \forall z, x \in K .
$$

In the following, we show that $F$ satisfies all the conditions of Theorem 2.3. 
Step 1. Condition (i) of Theorem 2.3 follows directly from assumption (3).

Step 2. By the definition of $F, F^{c}(z, x)=\{y \in X: \varphi(z, x, y)<0\}$ for all $z, x \in K$. By assumption (2), it is easy to see that $F^{c}(z, \cdot)$ is lower semicontinuous. We also know that $F^{c}(z, x)$ is convex from assumption (5). Hence condition (ii) of Theorem 2.3 holds.

Step 3. For any given $z \in K$ and any finite dimensional subspace $D$ of $X$ with $K_{D}=K \cap D \neq \emptyset$, consider the following problem: find $u \in K_{D}$ such that $\varphi(z, u, y) \geq 0$ for all $y \in K_{D}$. By assumptions (1)-(5), it follows from Lemma 3.5 that the above problem admits a nonempty, closed and convex solution set. That is to say that the set $\left\{x \in K_{D}: K_{D} \subset F(z, x)\right\}$ is nonempty, closed and convex. So condition (iii) of Theorem 2.3 is satisfied.

Step 4. Let $\left(x_{\alpha}, z_{\alpha}\right) \in K \times K,\left(x_{\alpha}, z_{\alpha}\right)$ converge to $(x, z) \in K \times K$ weakly, and $K \subset F\left(z_{\alpha}, x_{\alpha}\right)$ for all $\alpha$. It follows from the definition of $F$ that, for any $\alpha, \varphi\left(z_{\alpha}, x_{\alpha}, y\right) \geq 0$ for all $y \in K$. By Lemma 3.4, $\varphi\left(z_{\alpha}, y, x_{\alpha}\right) \leq 0$ for all $y \in K$. Assumption (6) implies that $\varphi(z, y, x) \leq 0$ for all $y \in K$. Again from Lemma 3.4, one has $\varphi(z, x, y) \geq 0$ for all $y \in K$. That is to say $K \subset F(z, x)$. So follows condition (iv) of Theorem 2.3. Based on Theorem 2.3, there exists $x^{*} \in K$ such that $K \subset F\left(x^{*}, x^{*}\right)$, i.e., $\varphi\left(x^{*}, x^{*}, y\right) \geq 0$ for all $y \in K$. The proof is complete.

Remark 3.7. In [10], a vectorial version of the problem in Theorem 3.6 was studied under $(S)_{+}$-conditions.

Remark 3.8. The problem in Theorem 3.6 includes as special cases some variational-like inequality problems studied in $[1,7]$.

From Theorem 3.6, we obtain the existence of solutions of the extended variational inequality in [8].

Corollary 3.9. Let $A: K \times K \rightarrow X^{*}$ (the dual space of $X$ ) be a nonlinear mapping satisfying the following conditions:

(i) for each $z \in K, A(z, \cdot)$ is pseudomonotone;

(ii) for each $x, w \in K,\langle A(\cdot, x), w\rangle$ is continuous with respect to the weak topology of $X$;

(iii) for each $z \in K$ and any finite dimensional subspace $D$ of $X$ with $K_{D} \neq \emptyset$, $A(z, \cdot): K_{D} \rightarrow X^{*}$ is continuous.

Then there exists $x^{*} \in K$ such that $\left\langle A\left(x^{*}, x^{*}\right), y-x^{*}\right\rangle \geq 0$ for all $y \in K$.

Proof. Define $\varphi: K \times K \times K \rightarrow R$ by

$$
\varphi(z, x, y)=\langle A(z, x), y-x\rangle \quad \forall x, y, z \in K .
$$

In the following, we shall show that all the assumptions of Theorem 3.6 are satisfied. The assumptions (3) and (5) of Theorem 3.6 follow directly from the 
definition of $\varphi$. Conditions (ii) and (iii) imply that assumptions (2) and (6) of Theorem 3.6 hold. For fixed $u \in K$, the pseudomonotonicity of $\varphi(u, \cdot, \cdot)$ follows from the pseudomonotonicity of $A(u, \cdot)$. Next, we show assumption (4) of Theorem 3.6 is satisfied. Let $u, x, y, z \in K$ such that $\varphi(u, x, y) \leq 0$ and $\varphi(u, x, z)<0$. It follows that $\langle A(u, x), y-x\rangle \leq 0$ and $\langle A(u, x), z-x\rangle<0$. Hence,

$$
\varphi(u, x, t y+(1-t) z)=\langle A(u, x), t y+(1-t) z-x\rangle<0 \quad \forall t \in(0,1) .
$$

Thus all the assumptions of Theorem 3.6 are satisfied. By Theorem 3.6, there exists $x^{*} \in K$ such that $\varphi\left(x^{*}, x^{*}, y\right) \geq 0$ for all $y \in K$, i.e., $\left\langle A\left(x^{*}, x^{*}\right), y-x^{*}\right\rangle \geq 0$ for all $y \in K$. The proof is complete.

Remark 3.10. Corollary 3.9 is a slight generalization of Theorem 2.1 in [8].

Acknowledgement. The authors would like to express their deep thanks to the anonymous referees for their helpful comments and suggestions. This work is supported by the Basic and Applied Research Projection of Sichuan Province (05JY029-009-1).

\section{References}

[1] Ansari, A. H., Siddiqi, A. H. and Yao, J. C., Generalized vector variationallike inequalities and their scalarizations. In: Vector Variational Inequalities and Vector Equilibria (ed.: F. Giannesi). Nonconvex Optim. Appl. 38. Dordrecht: Kluwer Acad. Publ. 2000, pp. 17 - 37.

[2] Ansari, A. H., Wong, N. C. and Yao, J. C., The existence of nonlinear inequalities. Appl. Math. Lett. 12 (1999)(5), 89 - 92.

[3] Berge, C., Topological Spaces, Including a Treatment of Multivalued Functions, Vector Spaces and Convexity (translated by E. M. Patterson). Edinburgh (Scotland): Olier \& Boyd 1963.

[4] Bianchi, M. and Schaible, S., Generalized monotone bifunctions and equilibrium problems. J. Optim. Theory Appl. 90 (1996)(1), 31 - 43.

[5] Blum, E. and W. Oettli, W., From optimization and variational inequalities to equilibrium problems. The Math. Student 63 (1994), 123 - 145.

[6] Chan, D. and Pang, J. S., The generalized quasivariational inequality problem. Math. Oper. Res. 7 (1982)(2), $211-222$.

[7] Chen, G. Y. and Hou, S. H., Existence of solutions for vector variational inequalities. In: Vector Variational Inequalities and Vector Equilibria (ed.: F. Giannessi). Nonconvex Optim. Appl. 38. Dordrecht: Kluwer 2000, pp. $73-86$.

[8] Chen, Y. Q., On the semi-monotone operator theory and applications. J. Math. Anal. Appl. 231 (1999), 177 - 192. 
[9] Di Bella, B., An existence theorem for a class of inclusions. Appl. Math. Lett. $13(2000)(3), 15-19$.

[10] Fang, Y. P. and Huang, N. J., Vector equilibrium type problems with $(S)_{+^{-}}$ conditions. Optimization 53 (2004)(3), $269-279$.

[11] Giannessi, F., Vector Variational Inequalities and Vector Equilibria. Dordrecht: Kluwer 2000.

[12] Giannessi, F. and Maugeri, A., Variational Inequalities and Network Equilibrium Problems. New York: Plenum Press 1995.

[13] Glicksberg, I., Further generalization of the Kakutani fixed point theorem with application to Nash equilibrium points. Proc. Amer. Math. Soc. 3 (1952), $170-174$.

[14] Guo, J. S. and Yao, J. C., Variational inequalities with nonmonotone operators. J. Optim. Theory Appl. 80 (1994)(1), 63 - 74.

[15] Hadjisavvas, N. and Schaible, S., Quasimonotone variational inequalities in Banach spaces. J. Optim. Theory Appl. 90 (1996)(1), 95 - 111.

[16] Harker, P. T. and Pang, J. S., Finite-dimensional variational and nonlinear complementarity problems: A survey of theory, algorithms and applications. Math. Programming 48 (1990), 161 - 220.

[17] Istrăescu, V. I., Fixed Point Theory: An Introduction. Dordrecht: Reidel Publishing Company 1981.

[18] Michael, E., Continuous selections I. Ann. of Math. 63 (1956), 361 - 382.

[19] Ricceri, B., Un theéorème d'existence pour les inéquations variationnelles. C. R. Acad. Sci. Paris Sér. I Math. 301 (1985), $885-888$.

Received July 18, 2004; revised December 29, 2004 